\section{BRADYCARDIA AND CARDIAC ARRHYTHMIA PRODUCED BY DEPRESSION OF CERTAIN OF THE FUNCTIONS OF THE HEART.}

BY JOHN HAY, M.D. VICT,, M.R.C.P. LOND., PHYSICIAN TO THE HOSPITAL FOR CONSUMPTION AND DISEASES OF THE CHEST, LIVERPOOL.

IN considering a case of heart disease the tendency is to lay stress rather on the nature and extent of the organic lesion and the character of the murmurs than on the actual power of the heart to do its work. The most important fact to be learnt is, of course, the area of cardiac response-the power of the heart to carry on its work and to meet stress. In the examination of an individual case, after having be present, the actual amount of hypertrophy and dilatation, the pathological nature of any lesion and the area of cardiac response, there is still a wide field for further investigation and one which ought in the next few years to yield a rich harvest in indications for treatment. I refer to the analysis of a heart's activity and the endeavour to discover in each PHYSICIAN TO THE STANLEY HOSPITAL, IIVERPOOL; ASSISTANT carefully formed an opinion as to the organic lesion, if one

contraction it finds the conducting power of the muscle able to transmit it at the normal rate. If from any cause the conductivity is depressed there is increased delay between the systole of the auricle and that of the ventricle, If the muscular fibres are in such a condition that they require, say, one second to regain their full conducting power, then a stimulus orjginating in the auricle every three-fifths of a second will not have allowed the fibres time to recover themselves and the passage of the stimulus will accordingly be delayed. If in such a case the auricular systole does not occur more frequently than every second then the delay between auricular and ventricular systole will be normal because the muscular fibres have been allowed full and adequate time for repair.

Clinically our method of estimating the conductivity in any case is by measuring the time between auricular and ventricular systole as recorded in the tracing of the venous pulse. For example, in the tracing Fig. 1 the space A between the two upright lines shows the time between the beginning of auricular systole $a$ and the ventricular systole as demonstrated by the pulse in the carotid artery, causing the small wave $c$ on the tracing. As the presphygmic time has not been taken into account this period $a c$ does not accurately represent the delay between auricular and ventricular systole, but as the error is relatively constant we may take this $a c$ interval as indicating the time required by the stimulus to pass along the muscle fibres from the auricle to the ventricle-in other words, to stand as our index of conductivity. Normally this $a c$ interval is oneFIG. 1.

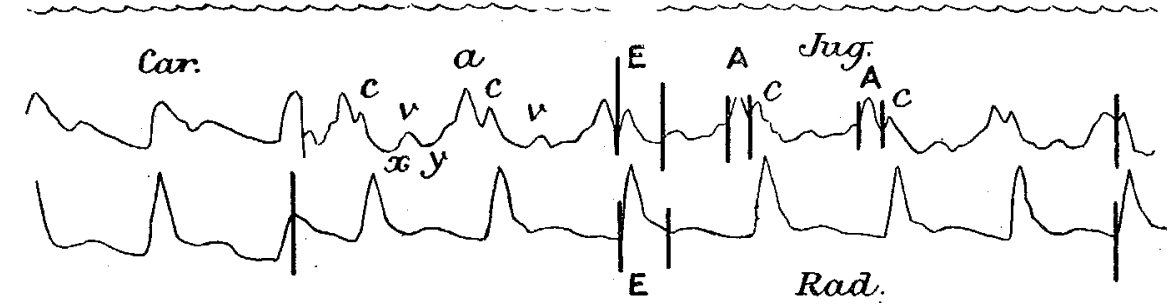

Tracing to show the normal or auricular type of venous pulse. The space E ndicates in all the tracings the period of ventricular systole during which the semilunar valves are open, the sphygmic period. The wave $a$, preceding the period $E$, can only be due systole during which the semilunar valves are open, the sphygmic period. The wave a, preceding the period $\mathrm{E}$, can only be due
to the systole of the right auricle. The fall $x$ is due to the diastole of the auricle causing a rush of blood out of the jugular vein to the systole of the right auricle. The fall $x$ is due to the diastole of the auricle causing a rush of blood out of the jugular vein
into the auricle, and the wave $c$ is due to the impact of the carotid pulse. The space $\mathrm{A}$ represents the $a c$ interval and indicates into the auricle, and the ware $c$ is due to the impact of the carotid pulse. The space A represents the $a c$ interval and indicates
the time taken by the stimulus to pass from the auricle to the venticle. It is normally one-fifth of a second. The time marker records one-fifth of a second. This applies to all the tracings.

case which of the special functions is chiefly at fault. The cardiac musculature possesses five great functionsrhythmicity or the power to produce its own rhythmic stimulus, excitability, conductivity or the power of conducting the stimulus from one muscle cell to another, con tractility, and tonicity. In any case of cardiac insufficiency or irregular action of the heart one or more of these functions must be either depressed or exalted. Our views of treatment will become enlarged and our therapeutic methods more scientific and efficient in so far as we are able to appreciate these variations. Such a knowledge can only be advanced by a careful study of individual cases. As our information becomes more complete and adequate cases which before had no relationship will be found to belong to the same group and to fail in the same essential function or functions although the coarse anatomical lesions be widely different.

The function which perhaps most easily lends itself to investigation and demonstration is that of conductivity, the conduction by the muscle cel's of the stimulus to contraction. We know that the reart does not contract simultaneously in all portions but that a peristaltic wave of contraction passes over it, starting "from the most automatically rhythmical tissue and travelling over the rest of the heart with varying speed." "The contraction of the heart signifies the passage of a contraction wave from one end of the heart to the other over muscular tissue of varying power of conductivity." The conduction is least in those portions of the musculature most nearly approaching the embryonic type of muscle, such as the fibres at the mouth of the great veins, in the auricular canal, and in the conus arteriosus. We know also that with each systole of the heart all the functions of the muscle fibres are temporarily abolished and that the process of recovery of function is relatively slow in cardiac muscle fibre. Normally the recovery in all the functions runs parallel, so that when sufficient "stimulus matter" has accumulated to initiate a

is to follow the function most impaired when he first came under observation was that of conductivity and my aim is to show the variations in this function while under observation and treatment; the effect of these variations on the pulse, producing sometimes a bradycardia, at others an arrhythmia; and the result of certain drugs in influencing the cardiac rhythm and the area of cardiac response. Later the function of excitability of the cardiac muscle became impajred and gave rise in its turn to a bradycardia and a condition of arrhythmia.

The patient, a man, aged 65 years, was admitted to the Stanley Hospital under the care of Dr. Whitford who very kindly transferred the case to me. The patient complained of stiffness in the right side over the liver and in the front of the chest. The family history was unimportant. $\mathrm{He}$ was on active service in India for nine and a half years and since then he has acted as dock labourer Previously to becoming a soldier his work was hard; latterly it has been easier though eatailing consider able exposure. In the use of alcohol he has always been moderate but for 50 years he has smoked heavily. $\mathrm{He}$ denies having had syphilis or gonorrhoea and he bas never suffered from typhoid fever or acute rheumatism. When in the East Indies he suffered for seven years from occasional severe attacks of "fever." For the last 12 years he has been liable to recurrent attacks of indigestion. For eight years he has been aware of the fact that his pulse was slow and for two and a half years he has experienced some degree of shortness of breath on exertion; this dyspncea has been steadily increasing. On Dec. 24th, 1904, he was attacked by gastritis and was "off work" for six weeks, during which time he was insensible for six days and six nights. Since this attack he has been at work, though steadily becoming less fit for it. He was admitted as an in-patient on June 8th, 1905. On admission he was found to be a moderately healthy looking man, contented, and well-nourished. There was no dropsy or jaundice. His 
temperature was normal and his lips were a little blue. There were pain and llatulence after food as well as some heartburn. The bowels were very constipated. The abdominal viscera were normal. There was nothing to indicate any disease of the kidneys. There was no marked emphysema. He has had a slight cough for years but with no expectoration. Dyspncea has gradually developed. He could only walk ten steps along the level at a time and could not go up stairs other than very slowly. He could lie flat in bed and sleep in that position. There has been, and is, a marked sense of giddiness accompanied by pain in the temples. For the past six weeks this pain has caused him to wish to shut his eyes. The pain became worse every evening about 9 o'clock; it continued through the night and became less severe in the morning, while during the day it was milder. For six weeks dizziness has been continuously present; this was less noticeable at night. He has had no sensation of impending faintness nor has there been any palpitation.

On June 24 th the patient's pulse was 32 per minute and regular in force and frequency. The blood pressure was 200 millimetres of mercury (systolic) and from 100 to 120 (diastolic). The arteries were thickened and somewhat tortuous. The apez beat was neither visible nor palpable. the right and five and a quarter inches to the left of the middle line, reaching above to the upper border of the third left costal cartilage and below to the seventh left rib. On auscultation the cardiac sounds were everywhere faint; at the apex there was a faint systolic bruit not conducted into the axilla; the first sound was inaudible, the second neither accentuated nor reduplicated. Both murmur and second sound were heard distinctly over the fourth left space towards the sternum. Over the aortic area a faint systolic murmur was audible with no alteration of the second sound. On carefully auscultating over and within the apex one occasionally heard during the diastole of the ventricle a faint whiff separated by a distinct interval from the second sound. A peculiar sequence of waves was observed in the veins of the neck. About the time of the radial pulse two waves occurred, one immediately following the other, and between every such a pair and the next pair of coupled waves there was a single wave. The faint whiff which was occasionally heard as noted above was observed to be synchronous in time with this single wave.

On the day after admission simultaneous tracings were taken of the radial and jugular pulses. In Fig. 2 we see that the auricle is beating twice as frequently as the ventricle, only every second systole of the auricle being able to initiate a ventricular systole. But the tracing shows more: it shows that the $a c$ interval is much longer than normal, that $A$ is two-fifths of a second instead of one-fifth, indicating delay in the passage or conduction of the stimulus from the auricle to the ventricle ; in other words, depression of the function of conductivity. The depression of conductivity is so marked that not only is there considerably increased delay in the passage of the stimulus from the auricle to the ventricle, as shown by the lengthened $a c$ interval, but there is also complete failure of the ventricle to respond to every other auricular systole. This failure is due to the fact that subsequently to the passage of the stimulus the conductivity is so much further depressed that the muscle fails entirely to transmit the second stimulus. The condition is one of heart block and is almost identical with the second of my two cases of bradycardia published in the British Medical Journal. ${ }^{1}$

The treatment consisted in light nutritious diet, mercurial purgation, and rest in bed. For a few days the patient was put on large doses of iodide of potassium and tabellæ trinitrini. It was then decided to see what effect the administration of atropine would have on the conductivity. He was accordingly, on June 17th, ordered tincture of belladonna was no change in the pulse the dose was increased to 15 minims, and five days later to 20 minims, every four hours. There was still no change in the pulse so the amount was further increased till on June 30th he was taking two drachms in the 24 hours. This large amount of atropine did not appear to have any effect on the pulse-rate or on the frequency of the auricular systole.

On July 4th the amount of the tincture of belladonna was increased to two drachms and 40 minims in the 24 hours.
Che patient felt somewhat better. His pupils were not markedly dilated, but there was some dryness of the throat. On the 7 th at 1.30 P.M. he was given one and a half drachms of the tincture of belladonna, followed in an hour by half a drachm and then later by ${ }_{1}^{3} 0$ ths of a grain of rtropine by the mouth. The pulse frequency did not increase beyond 36 per minute, but it was noticed that there was a slight irregularity (see tracings, Figs. 3, 5, 6) and also that the venous pulse in the neck became much larger and raore forcible. He received no more belladonna and on the $9 \mathrm{th}$ was ordered one grain of sodium nitrite every four hours. On the 11 th, after the patient had walked about, the pulserate was 40 , which dropped to from 32 to 36 under the influence of a short rest. It had regained its old rhythm and an $a c$ interval of two-fifths of a second. Tracings were taken similar to Fig. 2. The patient felt much better, the headache and giddiness had disappeared, and he was discharged "improved."

An examination of the tracings taken while the patient was under the influence of atropine reveals a most interesting condition. ${ }^{2}$ In Fig. 3 we have a greatly increased frequency of the auricular systole. The auricle is seen to be beating regularly with a frequency of from 85 to 90 beats per minute as compared with 67 beats per minute in Fig. 2 . The record of the radial pulse shows a peculiar arrhythmia, the cause of which is clear on analysing the tracings. With increased frequency of auricular beats the length of auricular diastole is diminished and the time allowed to the cardiac muscle to recover its conductivity is correspondingly shortened. In the tracings the result of this is seen by the effect on the $a c$ intervals. The ventricles, instead of responding to every second auricular systole as in Fig. 2, can now in a number of instances only respond to every third. Fig. 4 is a diagram built on the plan of Wenckebach and Mackenzie ; it demonstrates clearly what is taking place. The numbers indicate the $a c$ intervals which are drawn to scale and correspond to the numbers in Fig. 3 . We see that the $a c$ interval 1 is very prolonged, the time taken for the stimulus to pass from the auricle to the ventricle being three-fifths of a second; in 2 the $a c$ interval is shorter, in 3 shorter still, and in 4 it is practirally normal, being about one fifth of a second. The cause of this gradual shortening of the $a c$ interval is found in the fact that the ventricle has only been responding to every third auricular systole. The muscular fibres have for that reason had more time in which to recover their conductivity. This function has recovered to such a degree in 4 that following it the ventricle responds to the second auricular systole, with the result that again the $a c$ interval is abnormally prolorged because the fibres, though able to conduct the stimulus, were barely able to do so, having not had sufficient time to regain their normal power. The $a c$ intervals 6,7 , and 8 show the steady recovery of conductivity, the ventricle responding to every third auricular systole. This is a beautiful demonstration of the recovery of a function as the result of rest and also of the depression of that function as the result of activity. Figs. 5 and 6 were taken at the same time as Fig. 3 and are valuable as corroborative evidence of depressed conductivity. These tracings are good examples of the value of simultaneous records of the venous and radial pulses in explaining irregularities due to an abnormal condition of cardiac muscle.

Gibson has published a tracing in his paper on bradycardia showing a very similar condition-Case 1, p. 15. He has kindly allowed me to reproduce a copy of it (Fig. 7). On analysing this tracing we find that the rentricle is systole, then to every third, and that when the ventricle responds to the third auricular systole the $a c$ interval is thrice as long as when it responds to the fourth-the short extra time allowed the fibres for recovery, equal to the duration of an auricular systole and diastole, being sufficient to restore the conductivity to normal.

On Sept. 18th our patient was again admitted complaining chiefly of severe headaches and giddiness when walking about. Under sodium nitrite (one and a half grains thrice daily) he steadily improved. On the 26th there was still frontal and temporal headaches increased by the recumbent posture. There was troublesome insomnia and any exertion

2 Dr. W. T. Ritchie has very recently published in the Proceedings of the Royal Society of Edinburgh, vol. xxv. (p. 1085), an interesting case of complete heart-block with tracings, showing the effects of large doses of atropine. The auricular frequency was greatly increased, the
ventricular unaffected. 
FIG. 2.

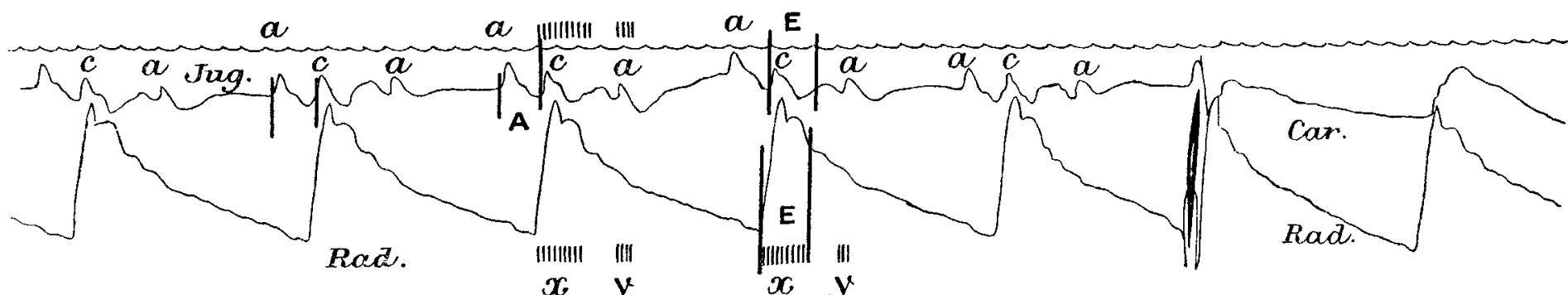

Simultaneous tracings of the radial and jugular pulses, showing that the $a c$ interval (space A) is twice the normal period (two-fifths of a second in place of one-fifth), indicating a delav in the passage of the stimulus from auricle to ventricle. It shows also that only every other systole of the auricle is followed by a systole of the ventricles. $x=$ murmur due to ventricular systole, mitral regurgitation; $y=$ murmur produced by auricular systole discharging blood into an incompletely filled ventricle. The time marking here represents one-fifth of a second.

FIG. 3.

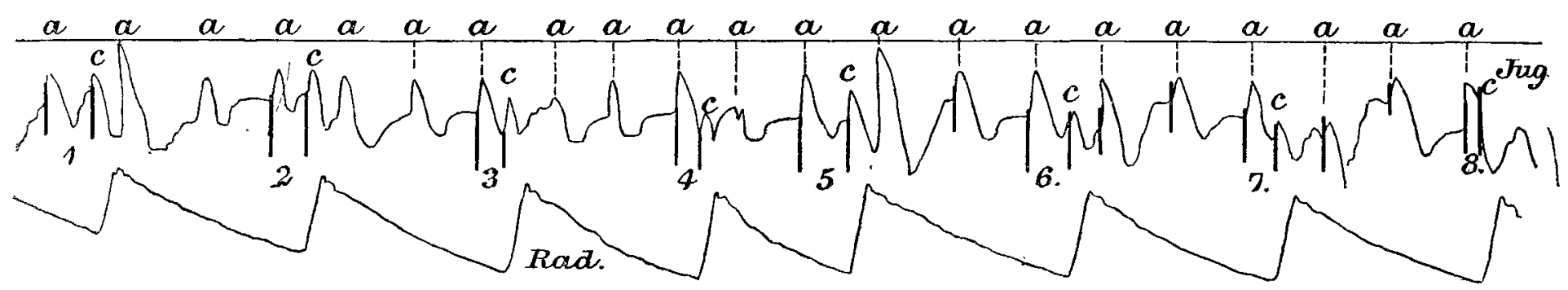

Tracing from the same patient after the administration of large doses of belladonna. The upper tracing is from the jugular vein and shows an increased frequency in auricular systoles. The numbers indicate the $a c$ intervals. In 1 and 5 it is lengthened to threefifths of a second; in 2,3 and 4 it gradually diminishes until in 4 it is almost normal ; in 6,7 , and 8 it again diminishes until in 8 it is quite normal. The radial pulse shows an arrhythmia. The pulses recorde 1 under the figures $2,3,4,6,7$, and 8 are longer than that under figure 5 , the reason being that in the former the ventricle is responding to every third auricular systole while in that under the figure 5 it responds to the second systole of the auricle.

FIG. 4.

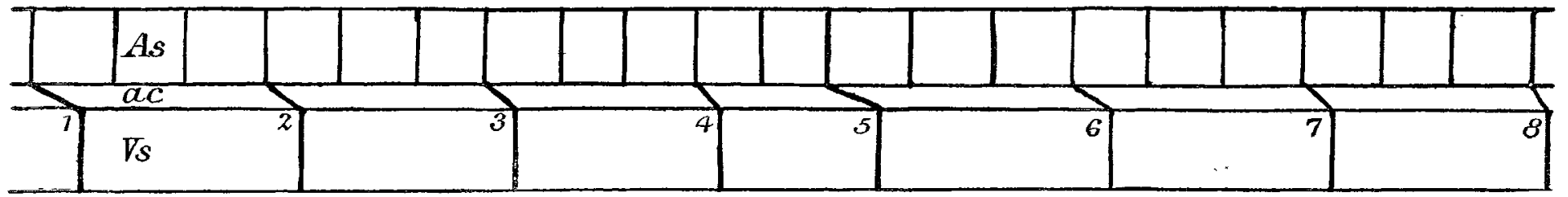

Diagram constructed from Fig. $3 A s=$ auricular systoles. $a c=$ the $a c$ interval numbered as in Fig. $3 . V s=$ Ventricular systoles. The diagram shows the cause of the irregularity of the radial pulse and demonstrates the recovery of the function of conductivity with rest.

FIG. 5.

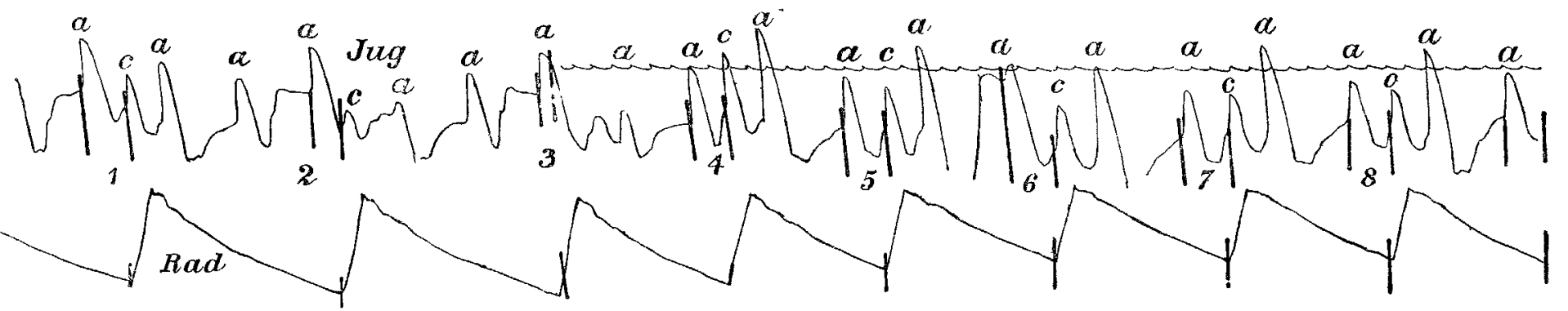

Tracing from the same patient at the same date as Fig. 3. The upper tracing is from the jugular vein and shows the increased frequency of auricular systole, also the steady recovery of conductivity as shown in the $a c$ intervals $1,2,3$, the recovery being possible in these instances only because the conducting fibres transmit every third stimulus and thus experience a longer period of rest. Throughout the remainder of the tracing the conducting fibres transmit every second stimulus and the $a c$ interval is lengthened, the intervals $4,5,6,7$, and 8 being a little over two-fifth of a second.

FIG. 6.
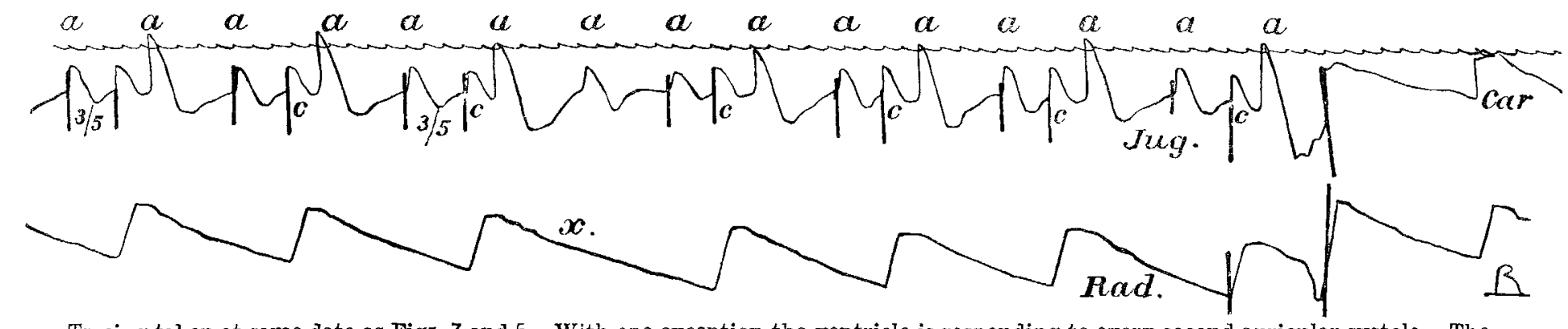

Tracing taken at same date as Figs. 3 and 5 . With one exception the ventricle is responding to every second auricular systole. The exception occurs in the middle of the tracing at $x$. where the ventricle is seen to refuse the second and respond to the third auricular systole. The tracing demonstrates marked depression of conductivitv and also a distinct improvement in the $\alpha c$ interval auricular systole. 
FrG. 7.

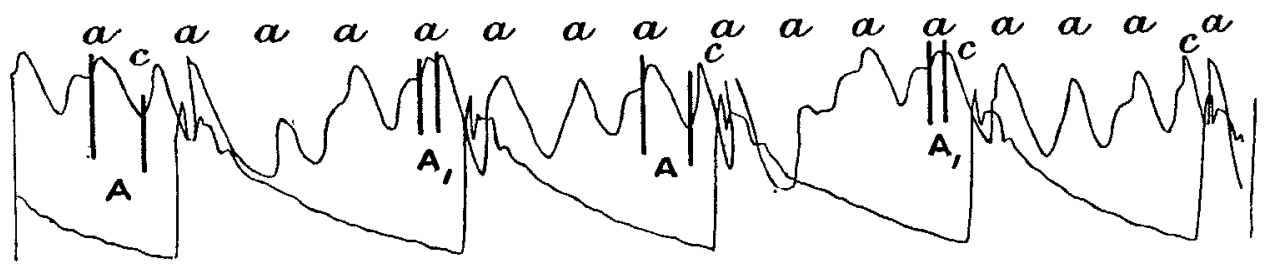

After Gibson. The upper tracing is from the jugular vein and shows the presence of numerous aurienlar systoles to every ventricular. The ventricle responds to every third or fourth auricular systole: when it responds to the third the $a c$ interval a is long. probably three-fifths of a second; when it responds to the fourth the $a c$ interval $A_{1}$ following is one-third the previous interval. The extra rest has been sufficient to restore the conductivity. The radial tracing shows depressions or little waves in its down stroke. caused by systoles of the left auricle.

FIG. 8.

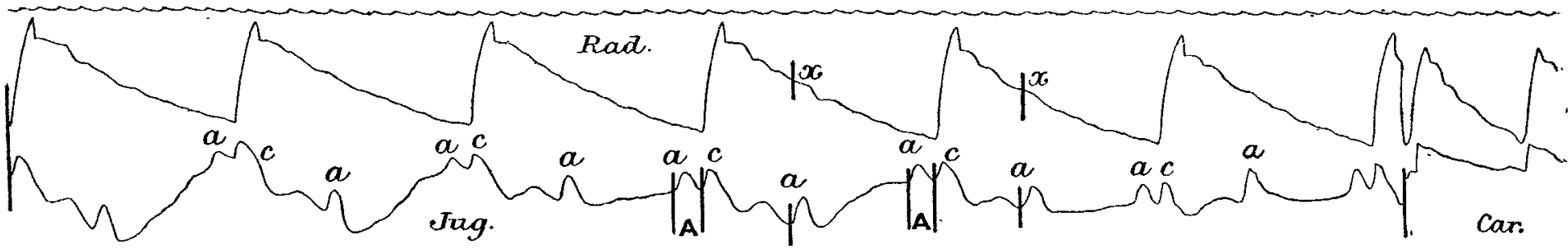

Tracing taken on Sept. 22nd, 1905. Shows heart block and also an $a$ c interval almost normal. The ventricle responds to every other auricular systole. The condition is one of brad veardia due to depression of excitability. At $x$ in the radial tracing one sees the slight rise followed by the dip caused by the systole of the left auricle. See also Fig. 7.

FIG. 9.

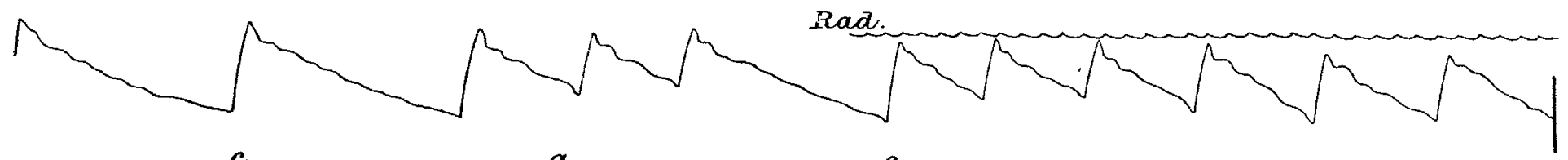

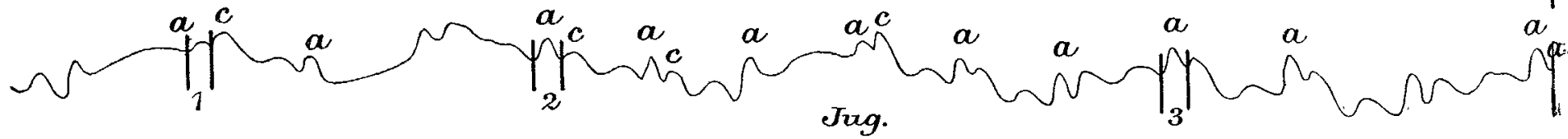

Tracing taken on the same date as Fig. 8. Shows the occasional dropping out of vent ricular svstoles with a normal conductivity, the $a c$ interval being about one-fifth of a second irrespective of the frequency of the pulse. Arrhythmia due to depression of excitability.

FIG. 10.

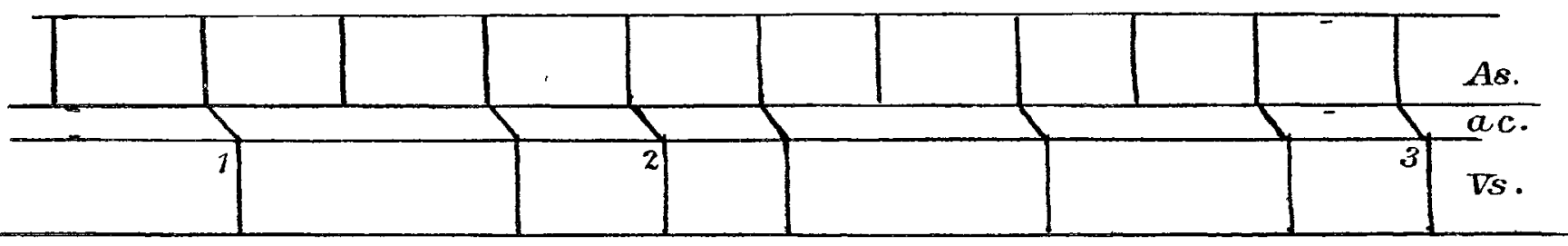

Diagram constructed from Fig. $9 . A s, a c, V s$ have the same significance as in Fig. 4 . Conductivity is shown to be normal and the $a c$ interval is the same throughout. Arrhythmia caused by depression of excitability.

FIG. 11.

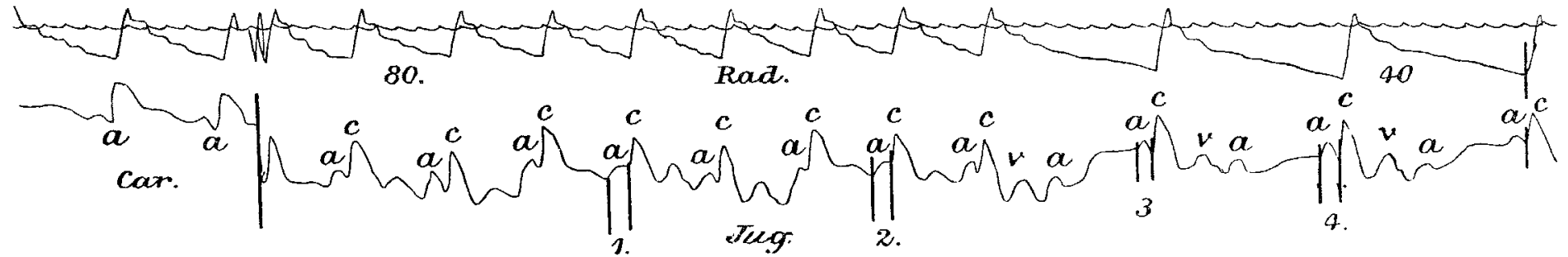

Tracing taken Oet. 19th showing sudden halving of the pulse frequency. No depression of conductivity; the $a c$ interral normal throughout. Depression of excitability is shown by the refusal of the ventricle, or possibly of the auricular canal, to respond to a

FIG. 12.

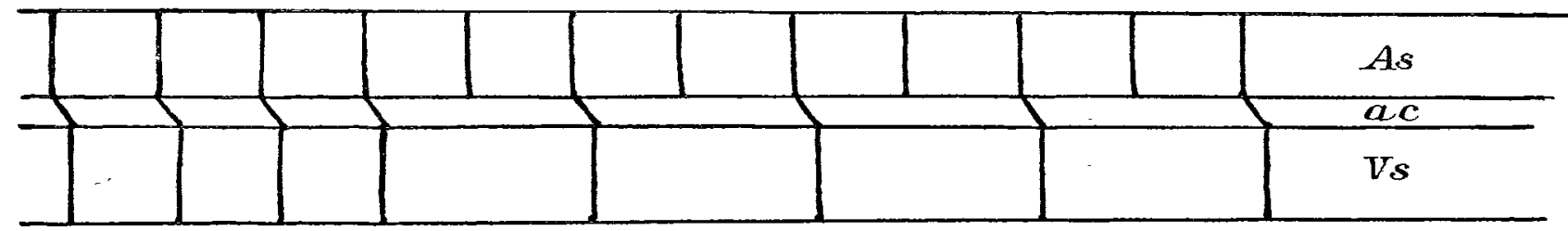

Diagram constructed from Fig. 11. As, ac, Is have the same significance as in Fig. 4 . Conductivity is shown to be normal as. the a $c$ interval. Bradycardia caused by depression of excitability. 
caused dyspnœa. The pulse varied greatly in frequency, it being sometimes 30 and sometimes 60 per minute and occassionally beat irregularly. At this time the more frequent rate was brought on by the patient lying down, by deep breathing, or by the excitement of having his pulse recorded. Mary traces were taken, of which Figs. 8, 9, and 11 are examples. On the 29th the pulse was of the same character. The beadache was much more severe though varying in intensity. When the headache was severe it was noted that the face was flushed. The patient was accordingly ordered adrenalin bydrochloride (1 in 1000) in 20 minim doses three times a day. From this point onward there was marked improvement.

On Oct. 19th it was noted that the headache was greatly ameliorated, the patient's colour was good, his appetite and digestion were excellent, and his area of cardiac response was greatly increased. On that date he was able to hurry up a hill without any discomfort. His pulse-rate varied, sometimes being 30 or 40 per minute and at other times between from 60 to 80 per minute. We examined him with the $x$ rays and were able to see that there was some dilatation of the descending portion of the arch of the aorta. At the beginning of the examination the heart frequency was 80 beats per minute and while we were looking at the shadow on the screen the frequency suddenly dropped too 40 , or half the previous rate. Fig. 11 shows a similar occurrence. During the long pauses which now occurred the ventricles were seen to remain motionless. We looked carefully to see any sign of auricular systole but failed. On Nov. 11th his condition was much the same, the pulse varying in a similar manner. Sir James Barr was present when the examination with the $x$ rays was made and suggested the administration of syrupus iodi tannici combined with the adrenalin hydrochloride.

Remarks.-These three tracings, Figs. 8, 9, and 11, show a remarkable change from the earlier ones and are well worth careful analysis. Looking at Fig. 8 we find that there is undoubted evidence of heart block, the ventricle only responding to every other systole of the auricle. At first sight the explanation which suggests itself is that we are still dealing with a depression of conductivity of such a degree that the conducting power has entirely recovered by the time that the second auricular systole occurs and the $a c$ interval is accordingly normal. The first auricular systole, however, finds the conductivity of the fibres in the process of recovery but still exhausted by the previous contraction and thus unable to transmit an impulse. A heart block occurs and every other ventricular systole drops out with the result that there is a bradycardia. Fig. 9 demonstrates that the above explanation is incorrect. There we see that the $a c$ interval is normal throughout whether the ventricle responds to each auricular systole or every second auricular systole. Fig. 11 shows the same condition but in a more marked degree.

On analysing a case of heart block due to depression of conductivity it is invariably found that the $a c$ interval varies according to the time given for rest-this is demonstrated in the tracings in Figs. 3, 5, 6, and 7. A clear appreciation of this fact compels us to dismiss the supposi. tion that the tracings Figs. 8, 9, and 11 indicate a depression of conductivity as the cause of this heart block. The $a c$ interval remains the same whether the period of rest is one or two seconds as in Fig. 9, or four-fifths or eight-fifths of a second as in Fig. 11. As a possible explanation it may be advanced that as a result of extrinsic nervous influences playing on the heart the conductivity is suddenly and seriously depressed to such an extent as to block completely the passage of every other stimulus. This is to me most improbable. A slight change in conductivity would be at once demonstrated by a lengthening of the $a c$ interval, not by a serious block. In the tracings Figs. 8, 9, and 11 it is, however, block or nothing. If, therefore, as is demonstrated, the conducting power is normal, to what must we ascribe the block? The power to initiate the stimulus is normal, the contractility of the musculature is normal, the tonicity is probably normal, and conductivity is practically normal. We are compelled, therefore, to conclude that the remaining function of excitability is the one impaired. Depression of excitability exists in this patient either in the fibres of the auricular canal or of the ventricle; the consequence is that a stimulus normally produced and normally conducted fails under certain conditions to obtain a response from these fibres.

The evidence, such as it is, favours the view that the depression of excitability is situated in the musculature of the ventricle. On carefully measuring the $a c$ intervals in Figs. 8 and 9 we find that their duration is a little over onefifth of a second whether the ventricle beats at 60 or 30 per minute. There is therefore a very slight depression of conductivity, not, however, of such a degree as to invalidate in any way the foregoing argument. Now, if through depression of excitability the auriculo-ventricular fibres had failed to respond to the stimulus reaching them they would certainly have attained the normal speed of conductivity by the time the next auricular stimulus reached them and the $a c$ interval would have been one-fifth of a second only. Figs, 3 and 5 show that, given a sufficient length of time, these fibres have the power of complete recovery. The inference is, therefore, that these fibres did respond to every stimulus, that they conducted it to the ventricle, and that there it failed on occasion to elicit a response. We have then a condition of heart block due to depression of the funcion of excitability. This function is here in $\mathbf{z}$ state of unstable equilibrium; mental excitement, alteration of posture, and deep breathing all tend to alter the extent of the depression and thus to vary the frequency of the ventricular systole.

Summary in conclusion.-We have in this case a condition of bradycardia due to heart block. When the patient was first seen the block was caused by depression of conductivity; later the conductivity became practically normal and the block which persisted was found to be caused by depression of excitability. No similar case has been recorded. The action of atropine was to increase the frequency of the stimulus production but had no influence on the power of conductivity. Sounds were heard during the diastolic phase of the ventricles; they were synchronous with the wave in the jugular vein caused by the systole of the right auricle. The assumption seems justifiable that the sounds were produced by the auricle in its systole. The depression of the conductivity and excitability of the myocardium is probably due to impaired metabolism, secondary to an insufficient blood-supply, the result of arterio-sclerotic changes in the coronary arteries. The excitability of the musculature was unstable, possibly as a result of nervous influences such as mental excitement.

Liverpool.

\section{ON THE RELIEF OF CERTAIN HEADACHES BY THE ADMINISTRATION OF ONE OF THE SALTS OF CALCIUM.}

By GEORGE W. ROSS, M.A., M.B. Toronto, M.R.O.S. ENG., L.R.C.P. LoND.,

PATHOLOGIST TO THE CITY OF IONDON HOSPITAL FOR DISEASES OF THE CHEST, LONDON, E.

(From the Pathological Laboratory of the Hospital.)

THERE occurs frequently in women, and occasionally in men, a type of headache which commonly presents the following characteristics: 1 . It is present and most severe on waking and tends to lessen in intensity or altogether disappear in from one to six hours. 2. It usually manifests itself as a dull heavy ache or as a frontal or temporal throbbing. Less often it is occipital, vertical, or unilateral. Infrequently it is neuralgic. 3. In its most typical form it is exceedingly chronic, often of several years' duration, and most intractable. It also exhibits itself as the common occasional headache to which many people are subject. 4. It is associated with a deficient coagulability of the blood. The subjects of the chronic form of this headache are usually of the lymphatic type. The expression is heavy and listless. The face is full and the eyes are often puffy. Some anæmia is the rule and it varies in intensity from a slight paleness to an actual chlorosis. The whole bearing exhibits mental and physical lassitude. Hereinafter the term "lymphatic type of headache" will be understood to refer to the above-described cephalalgia.

It is convenient to consider at this point certain symptoms commonly met with, and of the following several or many are usually found associated with the lymphatic headache. 